Gut, 1986, 27, 1377-1381

\title{
Wilson's disease: clinical presentation and use of prognostic index
}

\author{
HISHAM NAZER, R J EDE, A P MOWAT, AND ROGER WILliAMS \\ From the Department of Child Health and the Liver Unit, King's College Hospital and School of Medicine \\ and Dentistry, Denmark Hill, London
}

SUMmaRY As the results of treatment in Wilson's disease are so dependent on the stage at which penicillamine therapy is started, the antecedant history in 34 patients with Wilson's disease was analysed with particular respect to the earliest manifestations of the disease. Lethargy and anorexia $(70 \%)$ jaundice $(56 \%)$ and abdominal pain $(48 \%)$ were the commonest symptoms and less common were intellectual deterioration $(22 \%)$ and recurrent epistaxes $(22 \%)$. The duration of symptoms before diagnosis ranged from five days to three years (mean 10.5 months) and in only five of the patients was the diagnosis established before referral. Analysis of the physical signs at presentation showed hepatomegaly $(81 \%)$ and splenomegaly $(70 \%)$ to be common and the only signs which were significantly more common in the 13 fatal cases were jaundice and ascites. In three of these and in one other patient who survived the clinical course was exceptionally severe and was indistinguishable from fulminant hepatic failure. Based on the severity of abnormality of serum aspartate aminotransferase, bilirubin, and prothrombin time on admission a prognostic index was derived which enabled complete separation of fatal and nonfatal cases and when subsequently used in a further nine index cases correctly predicted the outcome. Two further cases found to have indices in the fatal category did well after liver transplantation, which needs to be considered as soon as the diagnosis is established in cases with such severe liver damage.

Initial symptoms in Wilson's disease are frequently non-specific and delay in diagnosis and institution of penicillamine therapy is all too common. This is particularly important because once signs of hepatic failure occur the response to chelation therapy is often disappointing. ${ }^{1}$ Indeed, episodes of jaundice earlier in childhood are usually attributed to infectious hepatitis ${ }^{2}$ and the possibility of underlying chronic liver disease is often overlooked. When presentation is with the classical neurological features of tremor, dysarthria and dystonia there is little difficulty in diagnosis but occasionally the only abnormality is a history of progressive intellectual deterioration which may not be brought to medical attention until a late stage. Similar delay in diagnosis may occur when presentation is with less common manifestations of the disease such as haemolytic anaemia,${ }^{3}$ haematuria ${ }^{4}$ amenorrhoea or musculoskeletal involvement. ${ }^{5}$ Moreover, it is rare for the diagnosis to be made, except in siblings of probands,

Address for correspondence: Dr Roger Williams. Liver Unit. King's College School of Medicine and Dentistry, Denmark Hill, London SE5 8RX, UK. Received for publication 28 February 1986. before significant liver or neurological damage has occurred.

In the present study we have retrospectively analysed the clinical and biochemical features present at diagnosis in 27 index cases and in seven asymptomatic siblings to determine which of the symptoms and signs are present early and likely to be overlooked. In addition we have attempted to define laboratory features which might allow the identification of patients with a poor prognosis at the time of presentation who might be suitable for hepatic transplantation. A prognostic index based on the abnormalities in liver function tests in these index cases has been applied prospectively in a further 11 index cases and in two of these hepatic transplantation was subsequently performed.
Methods
PATIENTS
DIAGNOSIS OF WILSON'S DISEASE AND DERIVATION OF PROGNOSTIC INDEX
Thirty four patients with Wilson's disease were seen 
in the Paediatric Department and Liver Unit of this hospital over the period January 1971 to August 1982. Diagnosis was based on the following criteria: serum caeruloplasmin $<0.2 \mathrm{~g} / \mathrm{l}$ (normal range 0.2 to $0.6 \mathrm{~g} / \mathrm{l})$ and/or urinary copper excretion $>1.25$ $\mu \mathrm{mol} / 24 \mathrm{~h}$ with an increased hepatic copper $(>300$ $\mu \mathrm{g} / \mathrm{g}$ dry liver). Other causes of liver disease such as chronic active hepatitis, hepatitis B virus infection, and $\alpha_{1}$-antitrypsin deficiency were excluded by appropriate investigations.

The severity of hepatic dysfunction on admission, as evidenced by the prolongation in prothrombin time, aspartate transferase activity and serum bilirubin was graded from $0-4$ as shown in the Table. A prognostic index based on the sum of these individual scores was calculated in all but one of the index cases for whom the biochemical data on admission was incomplete. Thus a maximum score of 12 was possible in those patients with the most severe derangement of liver function. This prognostic index was applied prospectively to a further 11 index cases admitted between August 1982 and April 1985.

\section{Results}

The mean age at presentation was 15 years (range 6-33 years) in the 27 symptomatic index cases (18 male and nine female patients) and 10.5 years (range 6 months-21 years) in the seven asymptomatic siblings (five male and two female) of the probands diagnosed by family screening. Thirteen $(48 \%)$ of the index cases died between one and 53 days of admission to this hospital, (five dying within the first five days of admission).

The duration of symptoms before referral of the 27 index cases ranged from five days to three years (mean 10.5 months). Lethargy and anorexia $(70 \%)$, jaundice $(56 \%)$ and abdominal pain $(48 \%)$ were the most common symptoms. A history of progressive intellectual deterioration was obtained in six $(22 \%)$ cases and the same number had a history of recurrent epistaxes. In three of the adult cases the presenting symptoms were predominantly neurological with tremor of one or both hands. One of these patients had been transiently jaundiced two years previously while another had had pain in the right knee for six months due to chondromalacia patellae.

The diagnosis of Wilson's disease had already been made in two children before referral. One had presented with haemolytic anaemia while the other was found to have asymptomatic hepatosplenomegaly when examined during a respiratory tract infection. The remaining 14 children (defined as age less than 15 years) were referred when liver disease was suspected because of the appearance of jaundice in the previous one to six weeks ( 11 children) or a raised aspartate transaminase detected in the previous two months (three children). In the latter group the initial features were a haemolytic anaemia starting six months earlier in one, haematuria and massive albuminuria of five months duration in the second, while the third patient had had malaise for five years, growth failure for three years and episodes of giddiness for one year. Of the 11 children referred with unexplained jaundice, five had been unwell for up to 18 months with lethargy and malaise but with the additional features of deteriorating school performance, abdominal pain, pallor, epistaxis, and fever each being present in one child. Non-specific abodminal pain of up to seven months duration was a dominant symptom in another three children with pallor, fever, and vomiting each being an additional feature in one child.

Non-specific malaise, nausea, vomiting, and abdominal pain were also presenting symptoms in five of the adult cases with unexplained jaundice and in three of these there was also a history of frequent epistaxes. In two adults and two of the 11 children who presented with jaundice the clinical picture was that of fulminant hepatic failure, with rapid onset of encephalopathy and death in three cases. The fourth case, a 16 year old boy, had a previous history of jaundice at the age of six years but had remained well until six weeks previously when he developed a

Table Derivation of prognostic index

\begin{tabular}{|c|c|c|c|c|c|}
\hline Score (in points) & 0 & $l$ & 2 & 3 & 4 \\
\hline \multicolumn{6}{|l|}{ Serum bilirubin* $(\mu \mathrm{mol} / \mathrm{l})$} \\
\hline (NR 3-20 $\mu \mathrm{mol} / \mathrm{l}$ ) & $<100$ & $100-150$ & $151-200$ & $201-300$ & $>300$ \\
\hline \multicolumn{6}{|l|}{ Serum aspartate* transferase (IU/I) } \\
\hline (NR 7-40 IU/l) & $<100$ & $100-150$ & $151-200$ & $201-300$ & $>3(0)$ \\
\hline Prolongation int prothrombin time (s) & $<4$ & $4-8$ & $9-12$ & $13-20$ & $>30$ \\
\hline
\end{tabular}

*Determined by Technicon SMAC 11 system.

+Determined by one stage Quick test standardised to international normalised ratio. 
frontal headache, diarrhoea, and lethargy but by the time of admission he had deteriorated to grade II encephalopathy.

Of the physical signs recorded at the time of diagnosis the most common were hepatomegaly $(81 \%)$, splenomegaly $(70 \%)$, Kayser-Fleischer rings by slit-lamp examination $(74 \%)$, jaundice $(63 \%)$ and pallor $(56 \%)$. Nearly half the patients had detectable ascites $(48 \%)$ while spider naevi were present in $30 \%$. Jaundice and ascites were significantly more common in the fatal cases, occurring in $92 \%$ and $85 \%$ respectively as compared with $29 \%$ and $14 \%$ of the survivors $(p<0 \cdot 001)$.

HAEMATOLOGICAL AND BIOCHEMICAL FINDINGS Ten patients were anaemic (haemoglobin $<10 \mathrm{~g} / \mathrm{dl}$ ) on admission while a further seven became anaemic during admission. In four cases this was caused by gastrointestinal haemorrhage while in six cases it was because of haemolysis with reticulocyte counts of up to $30 \%$.

Biochemical evidence of liver disease was found in all 27 cases. The serum bilirubin, aspartate transaminase and prothrombin time (normal range 12-13 seconds) at presentation were significantly more abnormal in those cases that subsequently died, the mean \pm SD being respectively $506 \pm 456$ $\mu \mathrm{mol} / \mathrm{l}, 710 \pm 635 \mathrm{IU} / \mathrm{l}$ and $49 \pm 35$ seconds prolonged as compared with $50 \pm 62 \mu \mathrm{mol} / \mathrm{l}, 93 \pm 78 \mathrm{IU} / \mathrm{l}$ and $4 \cdot 6 \pm 3.2$ seconds in the survivors $(p<0.005$, Wilcoxon's rank test). Use of the prognostic score in the 26 index cases for whom complete data were

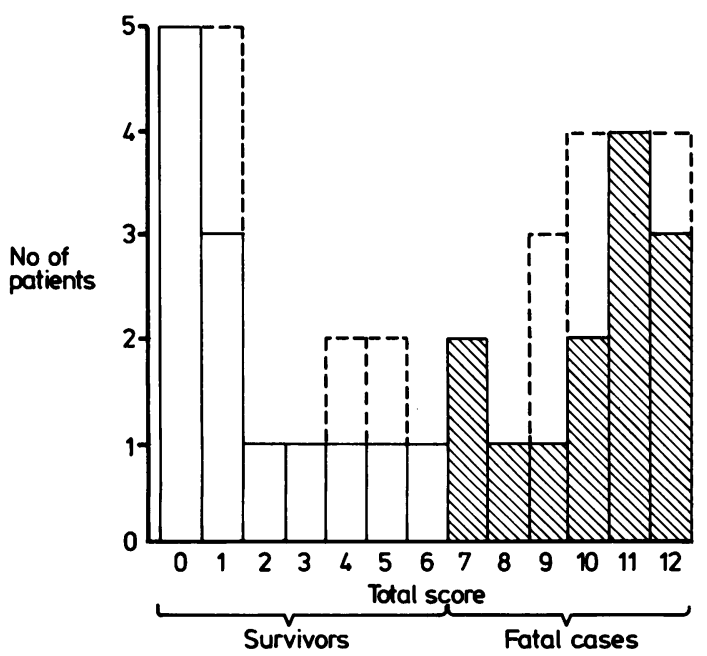

Fig. 1 Dotted lines represent the nine additional prospective cases, five of whom died (cross-hatched columns). available and in the nine additional cases revealed that all 17 cases with a score of six or less survived whereas the 18 patients with a score of seven or more on admission died (Fig. 1). A further two cases with scores of seven and eight received liver grafts and were therefore excluded from the analysis.

Serum immunoglobulins were estimated in 19 patients and in every case one or more of the classes was increased. Serum IgG was increased in 10 patients (mean $24 \cdot 2 \mathrm{~g} / \mathrm{l}$, range $15 \cdot 6-36 \mathrm{~g} / \mathrm{l}$ ), $\mathrm{IgA}$ in nine patients (mean $6.4 \mathrm{~g} / \mathrm{l}$, range $4 \cdot 7-8.6 \mathrm{~g} / \mathrm{l}$ ) and IgM in 12 patients (mean $6.6 \mathrm{~g} / \mathrm{l}$, range $2.4-9.3 \mathrm{~g} / \mathrm{l}$ ). Non-organ specific auto-antibodies (anti-nuclear, mitochondrial and smooth muscle), determined in 17 cases, were found in only one patient with a titre of $1: 40$ to smooth muscle antibodies.

Serum caeruloplasmin determined in 25 of the index cases, was low in $21(84 \%)$ cases, but normal in $4(16 \%)$ (median $0 \cdot 11 \mathrm{~g} / \mathrm{l}$, range $0-0.46 \mathrm{~g} / \mathrm{l})$. Urinary copper excretion was increased in $21(81 \%)$ of the 26 patients in whom it was determined (median $12 \cdot 2 \mu \mathrm{mol} / \mathrm{l}$, range $1 \cdot 3-145 \mu \mathrm{mol} / \mathrm{l}$ ). In none of the cases were both the serum caeruloplasmin and urinary copper excretion in the normal range. Serum copper, determined in 25 patients was normal in 11, increased in three and decreased in 11. The hepatic copper concentration was increased in all 14 cases in which it was determined with a median value of $681 \mu \mathrm{g} / \mathrm{g}$ dry liver (range 333-1780 $\mu \mathrm{g} / \mathrm{g}$, normal value $15-55 \mu \mathrm{g} / \mathrm{g}$ ). There was no significant difference in serum copper, serum caeruloplasmin, urinary copper excretion, or hepatic copper content between the fatal cases and those who survived.

Liver histology, obtained in 20 of the index cases, showed micronodular cirrhosis in 17 , chronic active hepatitis with moderate piecemeal necrosis, portal inflammatory infiltrate and bridging fibrosis in one, and diffuse fatty infiltration with nuclear vacuolation in another. In seven patients liver biopsy was not carried out because of excessive prolongation of prothrombin time.

\section{ASYMPTOMATIC SIBLINGS}

Seven siblings (three from the same family) were found to have Wilson's disease and their ages at diagnosis ranged from 6 months-20 years (mean 10.5 years). Hepatomegaly and Kayser-Fleischer rings were each detected in three patients while splenomegaly was detected in two. Biochemical tests of liver function were normal in all but one of the affected siblings in whom the serum aspartate aminotransferase was $155 \mathrm{IU} / \mathrm{l}$. In all seven patients haemoglobin, while cell counts, and platelet counts were within the normal range. Serum caeruloplasmin was low in four and urinary copper increased in 
six of the seven siblings. Percutaneous liver biopsy, done in four cases, showed minimal fatty changes with portal fibrosis in two cases and normal liver architecture in the remaining two. The liver copper content was increased in all four cases, (median $1180 \mu \mathrm{g} / \mathrm{g}$, range $800-1550 \mu \mathrm{g} / \mathrm{g}$ ).

Six of the siblings have remained asymptomatic since starting penicillamine therapy while the seventh developed Parkinsonian features and pain in the left elbow and both hips three months after starting this treatment. Radiological investigation of the hips revealed new bone formation arising from the lesser trochanters (Fig. 2a). Trientine dihydrochloride (TETA) was substituted for penicillamine and over the ensuing six months his symptoms slowly abated. A repeat radiograph eight months later showed considerable improvement with resorption and remodelling of the new bone (Fig. 2b).

\section{Discussion}

Although all 27 symptomatic cases showed some features of hepatic involvement at diagnosis these were rarely the first symptoms of the disease. Careful clinical evaluation, which showed abnormalities in five of the seven asymptomatic siblings, and standard biochemical testing of liver function might have led to earlier consideration of a liver disease. We had believed that the clinical and diagnostic features of Wilson's disease were already well known but with only five cases having had the correct diagnosis made before referral and four having documented haemolytic anaemia it is evident that the diagnosis is frequently overlooked. Particular difficulty in diagnosis occurs in patients presenting with fulminant hepatic failure in whom liver biopsy is precluded and the serum caeruloplasmin does not help in distinguishing Wilson's disease from other causes of fulminant hepatic failure. ${ }^{6}$ Urinary copper estimation may be helpful in such patients provided there is no renal failure. When caeruloplasmin and urinary copper excretion are equivocal determination of tissue copper concentration will usually be diagnostic although final histological
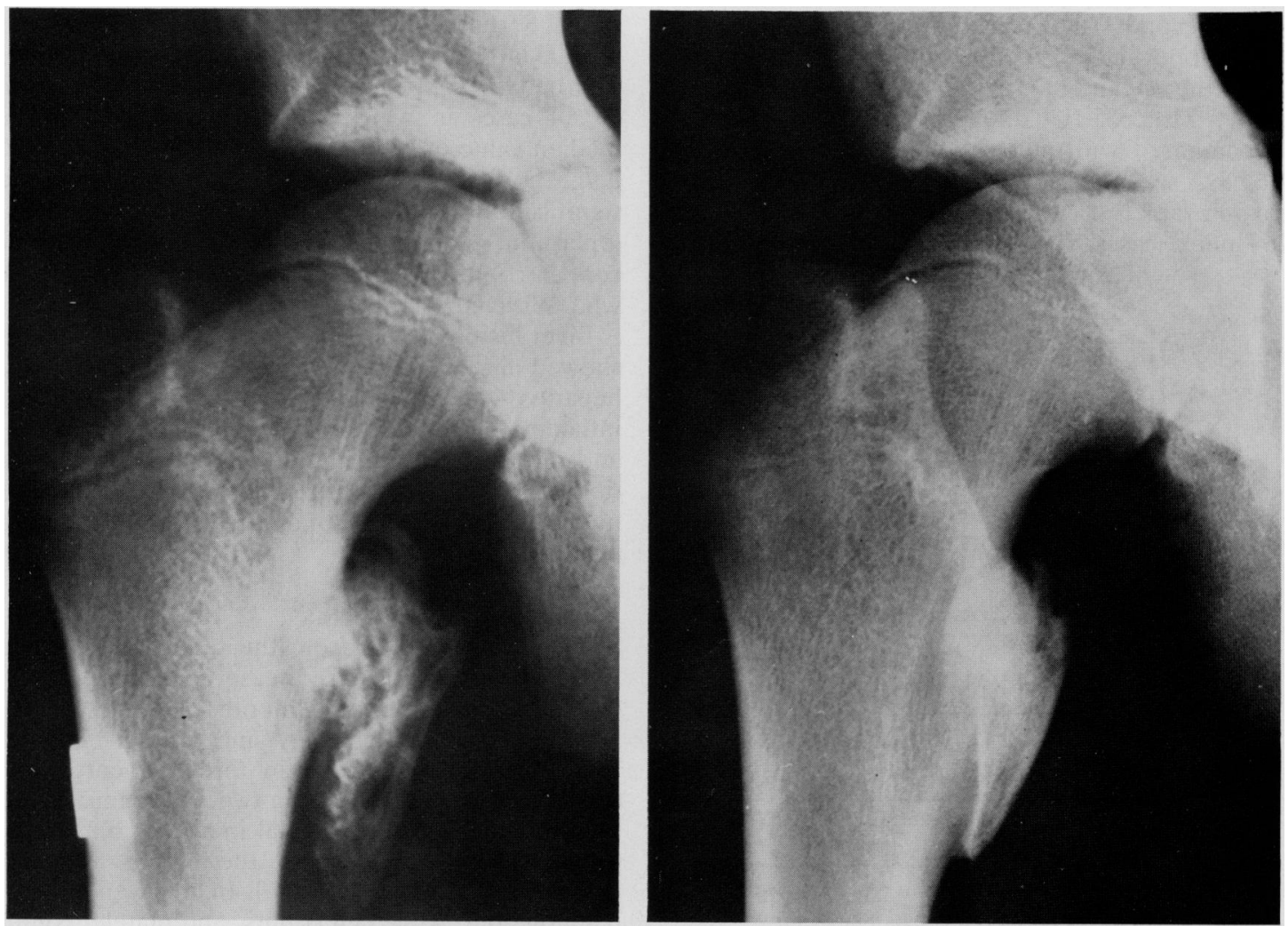

Fig. 2 (a) New bone formation over the lesser trochanter of the femur during treatment with D-penicillamine. (b) The same bone eight months later (following substitution of TETA) showing resorption and remodelling of the new bone. 
confirmation may only be possible, once recovery has occurred or at necropsy (as in one case in the present series). Using this approach it was possible to establish the correct diagnosis in every case in this series without recourse to expensive radioisotope uptake studies. ${ }^{7}$ It is significant that KayserFleischer rings, which are considered to be diagnostic in patients with a neurological presentation ${ }^{8}$ are frequently absent in those presenting with hepatological features of the disease ${ }^{9}$ and in the present series were absent on slit-lamp examination in $26 \%$ of cases.

The importance of screening the families of the index cases is shown by the diagnosis of Wilson's disease in seven asymptomatic siblings who remain well on long term chelation therapy. The case of the 17 year old boy who developed pain and stiffness in both hips within a few weeks of starting penicillamine is of particular interest. New bone formation is one of several skeletal abnormalities recognised in patients with Wilson's disease. In one series ${ }^{10} 33$ of 38 cases showed some evidence of bony abnormality which ranged from mild demineralisation in the majority to chondromalacia and osteoarthritis in 15 patients. The cause of these skeletal abnormalities is unknown but does not appear to be related to copper toxicity alone because copper loading in experimental animals does not produce skeletal changes. Golding and Walshe ${ }^{5}$ have suggested that these changes could be related to penicillamine therapy and the subsequent resorption and remodelling of the new bone during treatment with TETA appears to support this view. This chelating agent appears to be as effective as penicillamine in producing cupuresis ${ }^{11}$ but it is expensive, experience in its use is restricted, and supplies are limited. As the vast majority of patients are effectively treated with penicillamine, TETA should be used only in patients who develop severe side effects of penicillamine. The use of oral zinc sulphate which has been advocated $^{12} 1.3$ as an alternative treatment warrants further appraisal in our view.

With regard to the prognosis in Wilson's disease the presence of jaundice and ascites were the only clinical signs of prognostic value, being associated with a significantly higher mortality. Our prognostic index based on the scoring of liver function tests on admission, permitted complete separation between fatal cases and survivors and should therefore enable the likely outcome to be predicted in newly diagnosed cases, thus permitting earlier consideration of hepatic transplantation in those with a poor prognosis. Indeed, hepatic transplantation was successfully carried out in two such cases admitted to the Liver Unit in whom the prognostic index was seven and eight respectively (within the fatal range). Both these patients are alive and well having survived for three and a half years and one year respectively from the time of liver grafting. A third case with a prognostic index of seven died while awaiting a suitable donor organ. As liver grafting cures the underlying biochemical defect, chelation therapy is no longer necessary although lifelong immunosuppression will be required. In contrast all 17 patients with scores of six or less are now asymptomatic with normal liver function tests after the institution of chelation therapy. Early consideration of the diagnosis of Wilson's disease in patients with unexplained liver disease followed by prompt and appropriate treatment should enable the high mortality from this condition to be reduced significantly.

\section{References}

1 Nazer H, Ede RJ, Mowat AP, Williams R. Wilson's disease in childhood: variability of clinical presentation. Clin Paediatr 1983; 22: 755-7.

2 Silverberg M, Gellis SS. The liver in juvenile Wilson's disease. Paediatrics 1962; 30: 402-13.

3 McIntyre N, Clink HM, Levi AJ, Cumings JN, Sherlock S. Haemolytic anaemia in Wilson's disease. $N$ Engl J Med 1967; 276: 439.

4 Pellett H, Gallard L, Monier D, Ruitton-Ugliengo. Maladie de Wilson's a debut trompeur. Hematurie puis syndrome d'hypertension portale. Pediatrie 1965; 20: 472-3.

5 Golding DN, Walshe JM. Arthropathy of Wilson's disease. Study of clinical and radiological features in 32 patients. Ann Rheum Dis 1977; 36: 99-111.

$6 \mathrm{McCullough}$ AJ, Fleming CR, Thistle JL et al. Diagnosis of Wilson's disease presenting as fulminant hepatic failure. Gastroenterology 1983; 84: 161-7.

7 Sternlieb I, Scheinberg IH. The role of radiocopper in the diagnosis of Wilson's disease. Gastroenterology 1979; 77: 138-42.

8 Scheinberg I, Sternlieb I. Wilson's disease. Philadelphia. Saunders, 1984: 95.

9 Slovis TL, Dubois RS, Rodgerson DO et al. The varied manifestations of Wilson's disease. J Pediatr 1971; 78: 578-84.

10 Mindelzun R, Ekin M. Scheinberg IH, Sternleib I. Skeletal changes in Wilson's disease. A radiological study. Radiology 1970; 94: 127-32.

11 Walshe JM. Treatment of Wilson's disease with trientine (triethylene tetramine) dihydrochloride. Lancet 1982; 1: 643-7.

12 Brewer GJ, Gretchen MH, Prasad AS et al. Oral zinc therapy for Wilson's disease. Ann Intern Med 1983; 99: 314-20.

13 Deiss A. Treatment of Wilson's disease. Ann Intern Med 1983; 99: 398-400. 